\title{
Evaluation of PLC Channel Capacity and ABER Performances for OFDM-Based Two-Hop Relaying Transmission
}

\author{
Sana Ezzine, ${ }^{1,2}$ Fatma Abdelkefi, ${ }^{3}$ Jean Pierre Cances, ${ }^{1}$ \\ Vahid Meghdadi, ${ }^{1}$ and Ammar Bouallégue ${ }^{2}$ \\ ${ }^{1}$ CNRS, XLIM, UMR 7252, Université de Limoges, 87000 Limoges, France \\ ${ }^{2}$ Syscom Laboratory, University of Tunis El Manar, Tunis, Tunisia \\ ${ }^{3}$ Mediatron Laboratory, University of Carthage, Sup'Com, Tunis, Tunisia \\ Correspondence should be addressed to Sana Ezzine; sana.ezzine@gmail.com
}

Received 17 June 2016; Accepted 31 August 2016; Published 10 January 2017

Academic Editor: Rafael Pérez-Jiménez

Copyright (C) 2017 Sana Ezzine et al. This is an open access article distributed under the Creative Commons Attribution License, which permits unrestricted use, distribution, and reproduction in any medium, provided the original work is properly cited.

\begin{abstract}
Powerline network is recognized as a favorable infrastructure for Smart Grid to transmit information in the network thanks to its broad coverage and low cost deployment. The existing works are trying to improve and adapt transmission techniques to reduce Powerline Communication (PLC) channel attenuation and exploit the limited bandwidth to support high data rate over long distances. Two-hop relaying BroadBand PLC (BB-PLC) system, in which Orthogonal Frequency Division Multiplexing (OFDM) is used, is considered in this paper. We derive and compare the PLC channel capacity and the end-to-end Average BER (ABER) for OFDM-based direct link (DL) BB-PLC system and for OFDM-based two-hop relaying BB-PLC system for Amplify and Forward (AF) and Decode and Forward (DF) protocols. We analyze the improvements when we consider the direct link in a cooperative communication when the relay node only transmits the correctly decoded signal. Maximum ratio combining is employed at the destination node to detect the transmitted signal. In addition, in this paper, we highlight the impact of the relay location on the channel capacity and ABER for AF and DF transmission protocols. Moreover, an efficient use of the direct link was also investigated in this paper.
\end{abstract}

\section{Introduction}

Despite the fact that Powerline Communication (PLC) is still a very new medium for data transmission and that the international standards for Powerline Communication are still in the process of being developed and implemented, PLC is becoming a more current method for data transmission. Existing PLC stands as an important technology and communication architecture dedicated to Smart Grid (SG) deployment [1] thanks to its broad coverage and low cost deployment. The existing powerline infrastructure is employed in SG to transmit information together with the electric power. Furthermore, this solution can mainly guarantee high data rate, devices that can be easily connected, broad coverage in the SG, and low cost deployment contrarily to wireless technologies. Consequently, PLC has a core role in SG [2]. The main issue is that powerline is conceived for electrical energy transport and not for data communication.
It is known that the PLC channel exhibits multipath fading and frequency selective behaviors. Furthermore, its characteristics depend on the distance between the transmitter and the receiver [3, 4]. In [3], the authors have shown that, for high frequency signal propagation and long distances, PLC systems suffer from significant signal attenuation. Moreover, many researches have indicated that PLC channel depends on various parameters, such as network topology, connected loads, and location [5-7]. Additionally, PLC channel is a noisy channel and according to [8] it can be contaminated by a background noise or narrowband noise or impulse noise where narrowband noise is engendered by broadcast stations and the impulse noise is caused by switching power equipment in the network. The colored background noise is due to the superposition of many noise sources with low power and it spreads out in the allocated frequency band. Consequently, the data transmission for long transmission distances and for high frequencies in PLC systems is an interesting research 
topic. Orthogonal Frequency Division Multiplexing (OFDM) modulation technique facilitates of the powerline network for the routing of high-speed data thanks to its robustness and efficiency in facing frequency selective and fading channels $[9,10]$. The great advantage of OFDM is its plausibility in adjusting the transmission capacity to the channel propagation characteristics for each subcarrier in the frequency band.

Relay communications have been widely studied in wireless networks $[11,12]$ and it is proved that these techniques enhance spectrum efficiency and data rate. Consequently, many real schemata have recently been suggested to investigate relay communications in PLC systems for spectral efficiency, reliability, and data rate improvements [13-16]. The common protocols for relaying, such as Amplify and Forward (AF) and Decode and Forward (DF), have been proposed in PLC systems $[13,14,16]$. In $[13,14]$ authors have derived the capacity of direct link (DL) and AF-based Relay Aided (RA) over frequency selective PLC channels by restricting themselves to a single-carrier system. The authors proved that, compared to DL, RA in PLC system can lead to higher capacity. Furthermore, according to the simulation results, the highest capacity is achieved when the relay node is in the middle between the source and destination nodes.

In [16], authors studied the channel capacity of OFDM two-hop relaying PLC systems for Medium-Voltage (MV) powerlines for $\mathrm{AF}$ and $\mathrm{DF}$ protocols, over three wires, depending on the expectation of the noise at the destination node. Their researches have been performed for different MV topologies access networks for urban and rural environments [17]. The authors used the scattering-matrix (SM) method [18] to determine the channel responses resulting from cascade serial network modules. They studied channel capacity for OFDM two-hop relaying systems for two scenarios. In the first scenario, denoted by TDR (Time-Division Repeater), the relay retransmits, during the second time slot, the signal received from the source node during the first time slot by performing $\mathrm{AF}$ or $\mathrm{DF}$ protocol to the destination node. During the second scenario, denoted by TCR (Time-Division Cooperative Relaying), the source node sends signal to the destination and relay nodes in the first time slot. Then, the destination node receives, in the second time slot, signals from the source and the relay nodes. They suppose that the relay node decodes correctly the received signal from the source node in the DF protocol. Note that the authors did not perform a combination technique at the receiver to detect the transmitted signal and they did not consider the same allocated power at the relay node in the second time slot for AF and DF protocols. Numerical results, presented in [16], prove that the channel capacity over MV network of DF protocol is nearly identical to that reached for the AF protocol in the first scenario (TDR), but it is higher in the second scenario (TCR).

In this paper, we derive the channel capacity of a cooperative communication for OFDM-based BroadBand- (BB-) PLC system which operates in the high frequency band [2 MHz-30 MHz] based on the signal attenuation model for AF and DF protocols noted by PLC-AF and PLC-DF, respectively. We also derive the end-to-end Average Bit Error Rate (ABER) expression for OFDM-based two-hop relaying PLCAF and PLC-DF systems. To the extent of our knowledge, this is the first research work that is investigating this subject. Our main contributions, compared to the previously cited works, are mainly the following:

(i) To evaluate the contribution by using the direct link for two-hop relaying PLC systems based on the signal attenuation model, we considered two scenarios (with and without direct link) for OFDM-based two-hop relaying PLC-AF and PLC-DF systems. So, during the first scenario, the source node sends signal to the relay node during the first time slot; then the relay node proceeds to the AF or DF protocol on the received signal and transmits it in the second time slot to the destination node. In the second scenario, the source node transmits signal to the relay and destination nodes in the first time slot; then the relay node transmits the received signal according to $\mathrm{AF}$ or $\mathrm{DF}$ protocol in the second time slot to the destination node.

(ii) Since in the second scenario the destination node receives two signals after two time slots for OFDMbased two-hop relaying PLC-AF and PLC-DF systems, therefore it uses the maximum ratio combining (MRC) technique to recover the original signal. Then, we derive the expressions of MRC output signal at the destination node in the second scenario for $\mathrm{AF}$ and DF protocols.

(iii) Compared to [16], we suppose in the two investigated scenarios for OFDM-based two-hop relaying PLCDF system that the relay node can transmit the decoded signal to the destination node in the second time slot only if it decodes correctly the received signal from the source node.

(iv) Compared to $[13,14,16]$, we consider in this paper a summary for a cooperative communication in PLC systems where we propose to evaluate the channel capacity and ABER performances by varying the relay position for the two considered scenarios for OFDM-based two-hop relaying PLC-AF and PLC-DF systems.

Moreover we propose a solution based on data signal assignment indices permutation over the subcarriers to optimize the use of direct link in the second scenario for OFDM-based two-hop relaying PLC-AF and PLC-DF systems to improve the channel capacity and ABER performances. Furthermore, we assumed in this paper an Additive White Gaussian Noise (AWGN) model for the disturbance. We are aware that AWGN is a theoretical model for the noisy PLC channel [8] but it is sufficient here for simplicity of presentation and analysis.

The remainder of this paper is organized as follows. In Section 2, we briefly describe the PLC channel, the system model, and the studied protocols. In Section 3, we derive the channel capacity and ABER expressions for OFDM-based relaying systems for the AF and DF protocols for the two scenarios. Section 4 demonstrates the performance gain of 


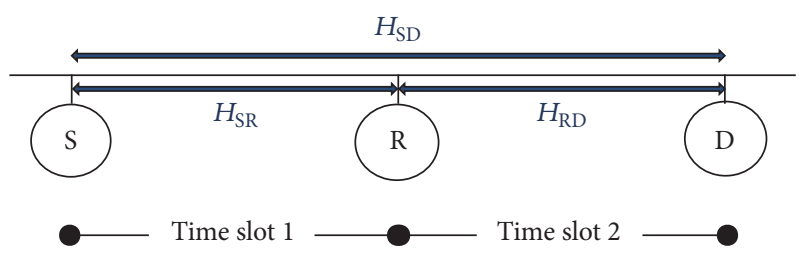

FIgURE 1: System model with two-hop relaying PLC network.

our approach, via simulation. Finally, Section 5 concludes the paper.

Notation. In the sequel, we will use the superscripts ${ }^{*}$ to denote element-wise conjugation and $\mathscr{E}[\cdot]$ to denote the expectation operator.

\section{PLC Channel and System Model}

2.1. PLC Channel. The literature proposes two categories of powerline channel models: the transmission line (TL) model $[19,20]$ and the multipath model $[3,4]$. In this paper, we exploited the theory of transmission lines or multipath model for modeling powerline channel in SG since it is an approach frequently considered in the literature. An approximate expression of the transfer function of PLC channel $H(f)$ implicates empirical parameters: $a_{0}, a_{1}$, and $k$ [3]. With reference to this model, $H(f)$ has the following expression:

$$
H(f)=e^{-\left(a_{0}+a_{1} f^{k}\right) d} e^{-j 2 \pi f \tau},
$$

where $e^{-\left(a_{0}+a_{1} f^{k}\right) d}$ denotes the overall attenuation and $e^{-j 2 \pi f \tau}$ is the delay of the wave and $\tau$ is the propagation delay. The resulting expression includes two parameters to evaluate the global attenuation. The parameter $a_{0}$ is the dependency of the attenuation with the length $d$ whilst the frequency selectivity is managed by $a_{1}$ and $n$. It is obvious that the attenuation becomes increases for the highest frequency and the longest distance between the transmitter and the receiver $[3,4]$.

2.2. System Model. In this paper, we investigate an OFDM system based on two-hop relaying system (see Figure 1), where we consider three nodes: the source, the relay, and the destination nodes denoted, respectively, by S, R, and D. The relay node is between the source and destination nodes in the powerline. Consequently, the source-relay distance $d_{\mathrm{SR}}$ and the relay-destination $d_{\mathrm{RD}}$ distance have a sum which is equal to the source-destination distance $d_{\mathrm{SD}}$. The source node sends data to the destination and is assisted by the relay node. We suppose that the relay node operates in half-duplex mode, so it cannot transmit and receive at the same time.

Since we used OFDM technique, the total system bandwidth, denoted by $B$, is split seamlessly into $N$ subcarriers. Accordingly, the bandwidth of each subcarrier is $\Delta f=$ $B / N$ and, on each subcarrier, the channel is supposed to be frequency-flat. In such architecture, the relay node receives the data from the source node and resends it to the destination node in two different time slots. All the subcarriers in the first time slot were served to the source for transmission.
TABLE 1: The investigated scenarios using TDMA.

\begin{tabular}{lcc}
\hline Scenarios & \multicolumn{2}{c}{ Time slot } \\
\hline 1 & $\mathrm{~S} \rightarrow \mathrm{R}$ & $\mathrm{R} \rightarrow \mathrm{D}$ \\
2 & $\mathrm{~S} \rightarrow \mathrm{R}, \mathrm{S} \rightarrow \mathrm{D}$ & $\mathrm{R} \rightarrow \mathrm{D}$ \\
\hline
\end{tabular}

During the second time slot, the relay node forwards the received signal to the destination node where AF or DF protocol is used and the total system bandwidth is used. For the AF protocol, the relay amplifies the received signal and transmits it to the destination node. For the DF protocol, the relay node must decode correctly the received signal from the source node to transmit the decoded signal to the destination node in the second time slot; otherwise it remains silent. For OFDM-based two-hop relaying following AF and DF in PLC systems, the transmission can be achieved through two scenarios summarized in Table 1 .

In scenario 1 , the source node transmits signal to the relay node during the first time slot. Then, the relay node amplifies and forwards or decodes and forwards the received signal from the source node to the destination node during the second time slot. In scenario 2, during the first time slot, the source node transmits signal to relay and destination nodes. In the second time slot, the relay node amplifies and forwards or decodes and forwards to the destination node the received signal.

The channel coefficients of the $n$th $(1 \leq n \leq N)$ subcarrier on $\mathrm{S} \rightarrow \mathrm{D}, \mathrm{S} \rightarrow \mathrm{R}$, and $\mathrm{R} \rightarrow \mathrm{D}$ links are denoted by $H_{\mathrm{SD}}[n]$, $H_{\mathrm{SR}}[n]$, and $H_{\mathrm{RD}}[n]$, respectively. Let $x[n]$ be the transmitted signal on the $n$th subcarrier. We assume that $\mathscr{E}[x[n]]=0$ and $\mathscr{E}\left[|x[n]|^{2}\right]=E_{x}[n]$. Since the PLC transmitted power is restricted by Electromagnetic Compatibility (EMC) regulations, so we maximize the entire transmitted data rate for a fixed transmitted power for all the subcarriers such that $E_{x}[n]=E_{x}, \forall n$, where $E_{x}$, is the maximum tolerated power level on subcarrier $n$. Let $y_{i j}[n]$ be the received signal on the $n$th subcarrier at node $j$ from node $i$, where $i \in\{\mathrm{S}, \mathrm{R}\}$ and $j \in\{\mathrm{R}, \mathrm{D}\}$. We denote by $n_{1, j}[n]$ and $n_{2, j}[n]$ the Additive White Gaussian Noise (AWGN) with zero mean and $\sigma^{2}[n]$ as variance which are received during the first and second time slots, respectively, at node $j$ with $j \in\{\mathrm{R}, \mathrm{D}\}$ on the $n$th subcarrier. We realize that AWGN is a simple representation for the PLC noise model but it is sufficient for the studied system (Figure 1) to analyze channel capacity and the ABER for PLC system according to the signal attenuation model. We suppose, for the rest of paper, that the noise variance is constant; that is, $\sigma^{2}[n]=\sigma^{2}, \forall n$.

2.3. Amplify and Forward (AF) Protocol. In this section, we illustrate the signal models for OFDM-based two-hop relaying PLC-AF systems in the two scenarios. For the first scenario, that is, without the direct link S-D, the received signal at the relay node during the first time slot at the $n$th subcarrier is expressed as

$$
y_{\mathrm{SR}}^{1, \mathrm{AF}}[n]=H_{\mathrm{SR}}[n] x[n]+n_{1, \mathrm{R}}[n] .
$$


Then, the relay node amplifies the received signal by a gain factor $\beta[n]=1 / \sqrt{E_{x}\left|H_{\mathrm{SR}}[n]\right|^{2}+\sigma^{2}}$ and forwards it to the destination node during the second time slot. Therefore, the received signal on the $n$th subcarrier during the second time slot at the destination node has the following expression:

$$
\begin{aligned}
y_{\mathrm{RD}}^{1, \mathrm{AF}}[n]= & \beta[n] H_{\mathrm{RD}}[n] \sqrt{E_{x}} y_{\mathrm{SR}}^{1, \mathrm{AF}}[n]+n_{2, \mathrm{D}}[n] \\
= & \sqrt{\frac{E_{x}}{E_{x}\left|H_{\mathrm{SR}}[n]\right|^{2}+\sigma^{2}}} H_{\mathrm{SR}}[n] H_{\mathrm{RD}}[n] x[n] \\
& +\widetilde{n}_{1, \mathrm{D}}[n],
\end{aligned}
$$

where the noise term $\widetilde{n}_{1, \mathrm{D}}[n]$ on the $n$th subcarrier is expressed as

$$
\begin{aligned}
\tilde{n}_{1, \mathrm{D}}[n]= & \sqrt{\frac{E_{x}}{E_{x}\left|H_{\mathrm{SR}}[n]\right|^{2}+\sigma^{2}}} H_{\mathrm{RD}}[n] n_{1, \mathrm{R}}[n] \\
& +n_{2, \mathrm{D}}[n] .
\end{aligned}
$$

In the second scenario (i.e., with direct link) the received signals during the first time slot on the $n$th subcarrier for the OFDM-based two-hop relaying PLC-AF systems at the relay node, denoted by $y_{\mathrm{SR}}^{2, \mathrm{AF}}[n]$, are defined by expression (2). The received signal from the source node at the destination node has the following expression:

$$
y_{\mathrm{SD}}^{2, \mathrm{AF}}[n]=H_{\mathrm{SD}}[n] x[n]+n_{1, \mathrm{D}}[n] .
$$

The relay node amplifies the received signal from the source node on the $n$th subcarrier by the factor $\beta[n]$ and transmits it to the destination node and this is achieved during the second time slot, $t$. So, the received signal during the second time slot at the destination node from the relay node, denoted by $y_{\mathrm{RD}}^{2, \mathrm{AF}}[n]$, is identical to $y_{\mathrm{RD}}^{1, \mathrm{AF}}[n]$ in (3). Since after two time slots the destination node $\mathrm{D}$ has received two versions of the original signal (i.e., from the relay and source nodes), therefore, it fuses the received signals from the source node $S$ and the relay node $\mathrm{R}$ in order to identify transmitted signal. This is optimally achieved by applying MRC technique [21]. It results in the fact that the combined signal at the MRC on the $n$th subcarrier can be expressed as

$$
y^{2, \mathrm{AF}}[n]=\omega_{1}[n] y_{\mathrm{RD}}[n]+\omega_{2}[n] y_{\mathrm{SD}}[n],
$$

where $\omega_{1}[n]$ and $\omega_{2}[n]$ correspond to the MRC coefficients on the $n$th subcarrier and they can be specified as $\omega_{1}[n]=$ $\beta[n] H_{\mathrm{SR}}^{*}[n] H_{\mathrm{RD}}^{*}[n] / \mathscr{E}\left[\left|\tilde{n}_{1, \mathrm{D}}[n]\right|^{2}\right]$ and $\omega_{2}[n]=H_{\mathrm{SD}}^{*}[n] / \sigma^{2}$.

2.4. Decode and Forward (DF) Protocol. In this section, we present the signal models for OFDM-based two-hop relaying PLC-DF system in the two scenarios. In the first scenario, the received signal from the source node during the first time slot on the $n$th subcarrier at the relay node is expressed as

$$
y_{\mathrm{SR}}^{1, \mathrm{DF}}[n]=H_{\mathrm{SR}}[n] x[n]+n_{1, \mathrm{R}}[n] .
$$

Then, the relay node decodes the received signal to recover the transmitted one from the source node during the first time slot. If the relay node is able to decode correctly the received signal, it forwards the decoded signal to the destination node during the second time slot. Otherwise, the relay node does not send any signals. Therefore, an ideal Cyclic Redundancy Check (CRC) code [22] should be incorporated so that the relay can decide even if the transmitted signal is correctly decoded or not. Then, the received signal at the destination node during the second time slot on the $n$th subcarrier has the following expression:

$$
y_{\mathrm{RD}}^{1, \mathrm{DF}}[n]=\alpha H_{\mathrm{RD}}[n] x[n]+n_{2, \mathrm{D}}[n] .
$$

Regarding parameter $\alpha$, it is fixed to 1 when the relay decodes correctly the received signal from the source node during the first time slot; otherwise $\alpha=0$.

In the second scenario and during the first time slot, the source node transmits signal to the relay and the destination nodes. After that, the received signal for OFDM-based twohop relaying PLC-DF system at the relay node on the $n$th subcarrier, $y_{\mathrm{SR}}^{2, \mathrm{DF}}[n]$, is identical to $y_{\mathrm{SR}}^{1, \mathrm{DF}}[n]$ in (7). We deduce the expression of the received signal at the destination node on the $n$th subcarrier as

$$
y_{\mathrm{SD}}^{2, \mathrm{DF}}[n]=H_{\mathrm{SD}}[n] x[n]+n_{1, \mathrm{D}}[n] .
$$

As previously outlined in the first scenario, if the relay node is able to decode correctly the received signal, it will forward the correctly decoded signal to the destination node during the second time slot on the $n$th subcarrier. Otherwise it will not send any signals or it will be idle. So, the received signal during the second time slot on the $n$th subcarrier at the destination node, denoted by $y_{2, \mathrm{RD}}^{2, \mathrm{DF}}[n]$, is identical to $y_{1, \mathrm{RD}}^{2, \mathrm{DF}}[n]$ in (8). The combined signal at the MRC on the $n$th subcarrier can be expressed as in (6) with weighting factors $\omega_{1}[n]=H_{\mathrm{SD}}^{*}[n] / \sigma^{2}$ and $\omega_{2}[n]=H_{\mathrm{RD}}^{*}[n] / \sigma^{2}$. Consequently, the combiner output signal at destination node is expressed as

$$
\begin{aligned}
y_{2, \mathrm{D}}^{2, \mathrm{DF}}[n]= & \frac{H_{\mathrm{SD}}^{*}[n]}{\sigma^{2}} y_{\mathrm{SD}}^{2, \mathrm{DF}}+\frac{H_{\mathrm{RD}}^{*}[n]}{\sigma^{2}} y_{2, \mathrm{RD}}^{2, \mathrm{DF}} \\
= & \frac{H_{\mathrm{SD}}^{*}[n]}{\sigma^{2}}\left(H_{\mathrm{SD}}[n] x[n]+n_{1, \mathrm{D}}[n]\right) \\
& +\frac{H_{\mathrm{RD}}^{*}[n]}{\sigma^{2}} \alpha\left(H_{\mathrm{RD}}[n] x[n]+n_{2, \mathrm{D}}[n]\right) \\
= & \left(\frac{\left|H_{\mathrm{SD}}\right|^{2}[n]+\alpha\left|H_{\mathrm{RD}}\right|^{2}[n]}{\sigma^{2}}\right) x[n] \\
& +\widetilde{n}_{2, \mathrm{D}}[n],
\end{aligned}
$$

where $\tilde{n}_{2, \mathrm{D}}[n]=\left(\left|H_{\mathrm{SD}}\right|^{*}[n] / \sigma^{2}\right) n_{1, \mathrm{D}}[n]+\left(\alpha H_{\mathrm{RD}}^{*}[n] /\right.$ $\left.\sigma^{2}\right) n_{2, \mathrm{D}}[n]$.

\section{Performance Analysis}

This section highlights the added value and advantages of cooperation through capacity and ABER metric for OFDMbased two-hop relaying PLC-AF and PLC-DF systems and the use of the direct link in this cooperative communication. 
3.1. System Capacity Evaluation. The capacity is an important metric which represents the maximum amount of information that can be transmitted per channel use. So, in this section, we analyze channel capacities in the two proposed scenarios in order to emphasize from derived equations, subsequently in Section 4, the contribution of the direct link (i.e., scenario 2). We suppose that the OFDM receivers have knowledge of the Channel State Information (CSI) and are perfectly synchronized. For OFDM systems, the total capacity corresponds to the sum of the achieved capacity on each subcarrier so it can be expressed as follows:

$$
C=\sum_{n=1}^{N} C[n]=\sum_{n=1}^{N} \Delta f \log _{2}\left(1+\gamma_{j}[n]\right) \quad(\text { bps }) \text {, }
$$

where $\gamma_{j}[n]$ is the effective Signal to Noise Ratio (SNR) at receiver $j$ on the $n$th subcarrier and $j \in\{\mathrm{R}, \mathrm{D}\}$.

3.1.1. Amplify and Forward (AF) Protocol. For the first scenario, we derive (from (11) which represents the received signal at the destination node) the effective SNR, that is, $\gamma_{\mathrm{D}}^{1, \mathrm{AF}}[n]$, at the destination node after two time slots on the $n$th subcarrier,

$$
\gamma_{\mathrm{D}}^{1, \mathrm{AF}}[n]=\frac{E_{x}}{\mathscr{E}\left[\left|\tilde{n}_{\mathrm{D}}\right|^{2}\right]}\left|\frac{\sqrt{E_{x}} H_{\mathrm{RD}}[n] H_{\mathrm{SR}}[n]}{\sqrt{E_{x}\left|H_{\mathrm{SR}}[n]\right|^{2}+\sigma^{2}}}\right|^{2},
$$

where $\mathscr{E}\left[\left|\widetilde{n}_{\mathrm{D}}\right|^{2}\right]=\left(E_{x}\left|H_{\mathrm{RD}}[n]\right|^{2} \sigma^{2} /\left(E_{x}\left|H_{\mathrm{SR}}[n]\right|^{2}+\sigma^{2}\right)\right)+\sigma^{2}$. We deduce the expression of $\gamma_{\mathrm{D}}^{1, \mathrm{AF}}[n]$ :

$$
\gamma_{\mathrm{D}}^{1, \mathrm{AF}}[n]=\frac{\gamma_{\mathrm{SR}}[n] \gamma_{\mathrm{RD}}[n]}{\gamma_{\mathrm{SR}}[n]+\gamma_{\mathrm{RD}}[n]+1},
$$

where $\gamma_{\mathrm{SR}}[n]=E_{x}\left|H_{\mathrm{SR}}[n]\right|^{2} / \sigma^{2}$ and $\gamma_{\mathrm{RD}}[n]=E_{x}\left|H_{\mathrm{RD}}[n]\right|^{2} /$ $\sigma^{2}$. It results in the fact that the channel capacity on the $n$th subcarrier can be expressed as

$$
C^{1, \mathrm{AF}}[n]=\frac{\Delta f}{2} \log _{2}\left(1+\gamma_{\mathrm{D}}^{1, \mathrm{AF}}[n]\right) .
$$

The factor $1 / 2$ is due to the fact that the information is transmitted to the destination node on two time slots.

In the second scenario, we can calculate from (6) and (13) the effective received SNR at the destination node on the $n$th subcarrier, denoted by $\gamma_{\mathrm{D}}^{2, \mathrm{AF}}[n]$, at the output of MRC. This effective SNR is the sum of the SNRs at the first and second time slots:

$$
\gamma_{\mathrm{D}}^{2, \mathrm{AF}}[n]=\gamma_{\mathrm{SD}}[n]+\gamma_{\mathrm{SRD}}^{\mathrm{AF}}[n]
$$

where $\gamma_{\mathrm{SD}}[n]=E_{x}\left|H_{\mathrm{SD}}[n]\right|^{2} / \sigma^{2}$ and $\gamma_{\mathrm{SRD}}^{\mathrm{AF}}[n]=\gamma_{\mathrm{D}}^{1, \mathrm{AF}}[n]$. It results in the fact that the channel capacity of the $n$th subcarrier can be calculated as

$$
C^{2, \mathrm{AF}}[n]=\frac{\Delta f}{2} \log _{2}\left(1+\gamma_{\mathrm{D}}^{2, \mathrm{AF}}[n]\right)
$$

3.1.2. Decode and Forward (DF) Protocol. In the first scenario, we define the maximum achievable capacity for the OFDMbased two-hop relaying PLC-DF system at the $n$th subcarrier as follows:

$$
\begin{aligned}
C^{1, \mathrm{DF}} & {[n] } \\
& =\frac{\Delta f}{2} \log _{2}\left(\min \left\{\left(1+\gamma_{\mathrm{SR}}[n]\right),\left(1+\gamma_{\mathrm{RD}}[n]\right)\right\}\right) .
\end{aligned}
$$

In the second scenario and after two time slots, the destination node receives signals from the source and the relay nodes in the first and second time slots, respectively. Then, as we already noted, the destination node uses MRC processing. Therefore, we define the maximum achievable capacity at the $n$th subcarrier as follows:

$$
\begin{aligned}
& C^{2, \mathrm{DF}}[n]=\frac{\Delta f}{2} \\
& \cdot \log _{2}\left(\min \left\{\left(1+\gamma_{\mathrm{SR}}[n]\right),\left(1+\gamma_{\mathrm{SD}}[n]+\gamma_{\mathrm{RD}}[n]\right)\right\}\right) .
\end{aligned}
$$

We note that the first term in (18) corresponds to the maximum rate at which the relay efficiently decodes the message sent from the source node. The second term, however, refers to the maximum rate based on which the destination node can reliably decode the original signal resulting from replicated transmissions from the source and the relay nodes.

3.2. ABER Performance Analysis. Another important metric is the Bit Error Rate (BER). Since the BER could be different for each subcarrier, we derive in this section the end-toend Average BER (ABER) expressions for OFDM-based two-hop relaying PLC-AF and PLC-DF systems for BPSK modulation at the destination node in the two previously detailed scenarios. We believe that this is the first work that proposes to evaluate the ABER for OFDM-based two-hop relaying PLC-AF and PLC-DF systems.

For an instantaneous SNR $\gamma[n]$ on the $n$th subcarrier at the destination node, the BER for BPSK modulation is expressed as

$$
P^{\gamma[n]}[n]=Q(\sqrt{2 \gamma[n]})
$$

where $Q(u)=(1 / \sqrt{2 \pi}) \int_{u}^{\infty} \exp \left(-t^{2} / 2\right) d t$ is the Gaussian $Q$-function [23]. Since the BER could be different for each subcarrier, then the end-to-end Average BER (ABER) of an OFDM PLC system at the destination node is defined as follows:

$$
P=\frac{1}{N} \sum_{n=1}^{N} P^{\gamma[n]}[n]
$$

3.2.1. Amplify and Forward. We derive in this section the expressions of ABER in the two scenarios (with and without S-D link), denoted by $P^{1, \mathrm{AF}}$ and $P^{2, \mathrm{AF}}$, respectively. By using 
the effective SNR at the destination node expressions previously calculated in (13) and (15), we deduce the expressions of $P^{1, \mathrm{AF}}$ and $P^{2, \mathrm{AF}}$ :

$$
\begin{aligned}
& P^{1, \mathrm{AF}}=\frac{1}{N} \sum_{n=1}^{N} \mathrm{Q}\left(\sqrt{2 \gamma_{\mathrm{D}}^{1, \mathrm{AF}}[n]}\right), \\
& P^{2, \mathrm{AF}}=\frac{1}{N} \sum_{n=1}^{N} \mathrm{Q}\left(\sqrt{2 \gamma_{\mathrm{D}}^{2, \mathrm{AF}}[n]}\right) .
\end{aligned}
$$

3.2.2. Decode and Forward. In this paragraph, we derive analytic expression for ABER at the destination node for the two scenarios, denoted by $P^{1, \mathrm{DF}}$ and $P^{2, \mathrm{DF}}$.

In the first scenario, that is, without S-D link, the relay node transmits to the destination node until it correctly decodes the received signal from the source node. Otherwise, it does not send any signals. So, it decodes the received signal $y_{\mathrm{SR}}[n]$ and judges whether it is correctly decoded or not by using CRC code during the first time slot. Thus, the relay node $\mathrm{R}$ may, or may not, correctly decode the received signal. Clearly, at $\mathrm{R}$, an error event $e_{\mathrm{R}}$ and a non-error event $\bar{e}_{\mathrm{R}}$ will affect the recovered signal at the destination node $\mathrm{D}$. We denote by $P\left(e_{\mathrm{R}}\right)$ the probability of an error event $e_{\mathrm{R}}$ at the relay node $\mathrm{R}$ and by $P\left(\bar{e}_{\mathrm{R}}\right)$ its complement, that is, the probability of a correct decision at the relay node $\mathrm{R}$ such that $P\left(\bar{e}_{\mathrm{R}}\right)=1-$ $P\left(e_{\mathrm{R}}\right)$. The destination node receives a signal when the relay node correctly decodes the received signal and this event has an occurrence probability equal to $P\left(\bar{e}_{\mathrm{R}}\right)$. Otherwise the destination node did not receive any signals from the relay node when this latter could not correctly decode the received signal with the probability $P\left(e_{\mathrm{R}}\right)$. This event has an occurrence probability equal to 0.5 . Consequently, the error probability at the destination node $\mathrm{D}$ has the following expression:

$$
P\left(e_{\mathrm{D}}\right)=P\left(\bar{e}_{\mathrm{R}}\right) P\left(e_{\mathrm{D}} \mid \bar{e}_{\mathrm{R}}\right)+0.5 P\left(e_{\mathrm{R}}\right),
$$

where $P\left(e_{\mathrm{D}} \mid \bar{e}_{\mathrm{R}}\right)$ is the error probability at the destination node $\mathrm{D}$ when the relay node $\mathrm{R}$ decodes correctly the received signal. In the case of a BPSK modulation, the error probability at the relay node $\mathrm{R}$ on the $n$th subcarrier has the following expression:

$$
P\left(e_{\mathrm{R}}\right)[n]=Q\left(\sqrt{2 \gamma_{\mathrm{SR}}[n]}\right) .
$$

Recalling that the probability of the correct decision at the relay node $\mathrm{R}$ on the $n$th subcarrier $P\left(\bar{e}_{\mathrm{R}}\right)[n]$ is equal to $1-P\left(e_{\mathrm{R}}\right)[n]$, then it is expressed as

$$
P\left(\bar{e}_{\mathrm{R}}\right)[n]=1-Q\left(\sqrt{2 \gamma_{\mathrm{SR}}[n]}\right) .
$$

The error probability at the destination node D on the $n$th subcarrier when the estimation at the relay node $\mathrm{R}$ on the $n$th subcarrier is correct is given by

$$
P\left(e_{\mathrm{D}} \mid \bar{e}_{\mathrm{R}}\right)[n]=\mathrm{Q}\left(\sqrt{2 \gamma_{\mathrm{RD}}[n]}\right) .
$$

It results in the fact that $P\left(e_{\mathrm{D}}\right)[n]$ is defined as

$$
\begin{aligned}
P\left(e_{\mathrm{D}}\right)[n]= & \left(1-Q\left(\sqrt{2 \gamma_{\mathrm{SR}}[n]}\right)\right) Q\left(\sqrt{2 \gamma_{\mathrm{RD}}[n]}\right) \\
& +0.5 \mathrm{Q}\left(\sqrt{2 \gamma_{\mathrm{SR}}[n]}\right) \\
= & \left(\sqrt{2 \gamma_{\mathrm{RD}}[n]}\right) \\
& -Q\left(\sqrt{2 \gamma_{\mathrm{SR}}[n]}\right) Q\left(\sqrt{2 \gamma_{\mathrm{RD}}[n]}\right) \\
& +0.5 \mathrm{Q}\left(\sqrt{2 \gamma_{\mathrm{SR}}[n]}\right) \\
\approx & Q\left(\sqrt{2 \gamma_{\mathrm{RD}}[n]}\right)+0.5 Q\left(\sqrt{2 \gamma_{\mathrm{SR}}[n]}\right)
\end{aligned}
$$

and $P^{1, \mathrm{DF}}$ is deduced as

$$
\begin{aligned}
& P^{1, \mathrm{DF}} \\
& \quad=\frac{1}{N} \sum_{n=1}^{N}\left(\mathrm{Q}\left(\sqrt{2 \gamma_{\mathrm{RD}}[n]}\right)+0.5 \mathrm{Q}\left(\sqrt{2 \gamma_{\mathrm{SR}}[n]}\right)\right) .
\end{aligned}
$$

In the second scenario, that is, with S-D link, the destination node receives signals from the source node in the first time slot and from the relay node in the second time slot only when it can correctly decode the received signal from the source node. If the relay recovers correctly the received signal, which occurs with a probability equal to $P\left(\bar{e}_{\mathrm{R}}\right)$, then the effective SNR at the destination node is deduced from (10); that is, $\gamma_{\mathrm{D}}^{2, \mathrm{DF}}=\gamma_{\mathrm{D}}^{\mathrm{MRC}, \mathrm{DF}}=\gamma_{\mathrm{SD}}^{2, \mathrm{DF}}+\gamma_{\mathrm{RD}}^{2, \mathrm{DF}}$. Otherwise, the effective SNR is equal to the SNR in the direct link; that is, $\gamma_{\mathrm{D}}^{2, \mathrm{DF}}=\gamma_{\mathrm{SD}}^{2, \mathrm{DF}}$. This latter event occurs with a probability equal to $P\left(e_{\mathrm{R}}\right)$. It results in the fact that $P\left(e_{\mathrm{D}}\right)[n]$ is expressed as

$$
\begin{aligned}
P\left(e_{\mathrm{D}}\right)[n] & \\
= & \left(1-Q\left(\sqrt{2 \gamma_{\mathrm{SR}}[n]}\right)\right) Q\left(\sqrt{2\left(\gamma_{\mathrm{SD}}^{2, \mathrm{DF}}+\gamma_{\mathrm{RD}}^{2, \mathrm{DF}}\right)}\right) \\
& +Q\left(\sqrt{2 \gamma_{\mathrm{SR}}[n]}\right) Q\left(\sqrt{2 \gamma_{\mathrm{SD}}[n]}\right) \\
\approx & \left(1-\mathrm{Q}\left(\sqrt{2 \gamma_{\mathrm{SR}}[n]}\right)\right) Q\left(\sqrt{2\left(\gamma_{\mathrm{SD}}^{2, \mathrm{DF}}+\gamma_{\mathrm{RD}}^{2, \mathrm{DF}}\right)}\right) .
\end{aligned}
$$

And $P_{2}^{2, D F}$ is deduced as

$$
\begin{aligned}
& P^{2, \mathrm{DF}}= \frac{1}{N} \sum_{n=1}^{N}\left(1-Q\left(\sqrt{2 \gamma_{\mathrm{SR}}[n]}\right)\right) \\
& \cdot Q\left(\sqrt{2\left(\gamma_{\mathrm{SD}}^{2, \mathrm{DF}}+\gamma_{\mathrm{RD}}^{2, \mathrm{DF}}\right)}\right) .
\end{aligned}
$$

\section{Simulation Results}

To afford quantitative testing of the end-to-end channel capacity and ABER of the different performed scenarios (i.e., with and without S-D link) for AF and DF protocols, we 
TABLE 2: Simulation parameters.

\begin{tabular}{lc}
\hline Bandwidth $B$ & {$[1.6 \mathrm{MHz}-30 \mathrm{MHz}]$} \\
Distance between two nodes " $d$ " & $100,200,300 \mathrm{~m}$ \\
Subcarrier number " $N$ " & 1024 \\
Maximum power on each subcarrier " $P$ ” & $-60 \mathrm{dBm} / \mathrm{Hz}$ \\
Subcarrier bandwidth " $\Delta f$ " & $27.734 \mathrm{kHz}$ \\
\hline
\end{tabular}

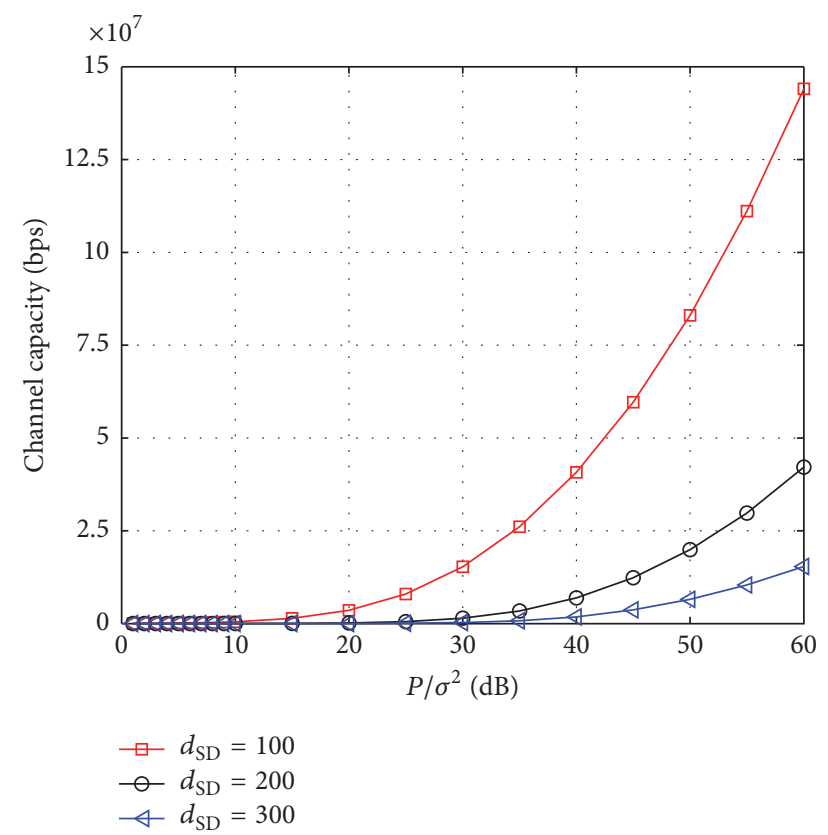

FIGURE 2: Channel capacity for OFDM-based DL-PLC systems.

contemplate a PLC line topology similar to the one depicted in Figure 1 which represents a linear three-node arrangement of PLC-SN network. The simulation parameters are provided in Table 2.

In this paper, the experimental values $a_{0}, a_{1}$, and $k$ for the signal attenuation model for different transmission distances are defined in [3].

4.1. Numerical Results. As previously stated, the signal attenuation in PLC systems is significant for long transmission distances. So, to show this impact on channel capacity, this effect is illustrated in Figure 2 in the case of an OFDM-based DL-PLC system. As it can be clearly observed, the channel capacity decreases dramatically when the source-destination distance increases and when $P / \sigma^{2}$ decreases. This result is obvious since the channel capacity is proportional to the logarithm of the effective SNR at the destination node given by (11).

Figure 3 shows the channel capacity for OFDM-based two-hop relaying PLC-AF system in the two previously detailed scenarios for different relay location ratios $d_{\mathrm{SR}} / d_{\mathrm{SD}}$ when communication distance $d_{\mathrm{SD}}$ is equal to $100 \mathrm{~m}, 200 \mathrm{~m}$, and $300 \mathrm{~m}$ and $P / \sigma^{2}=20 \mathrm{~dB}$. We note that the highest system capacity is achieved when the relay node is betwixt the source and destination nodes. This can be explained by the fact that

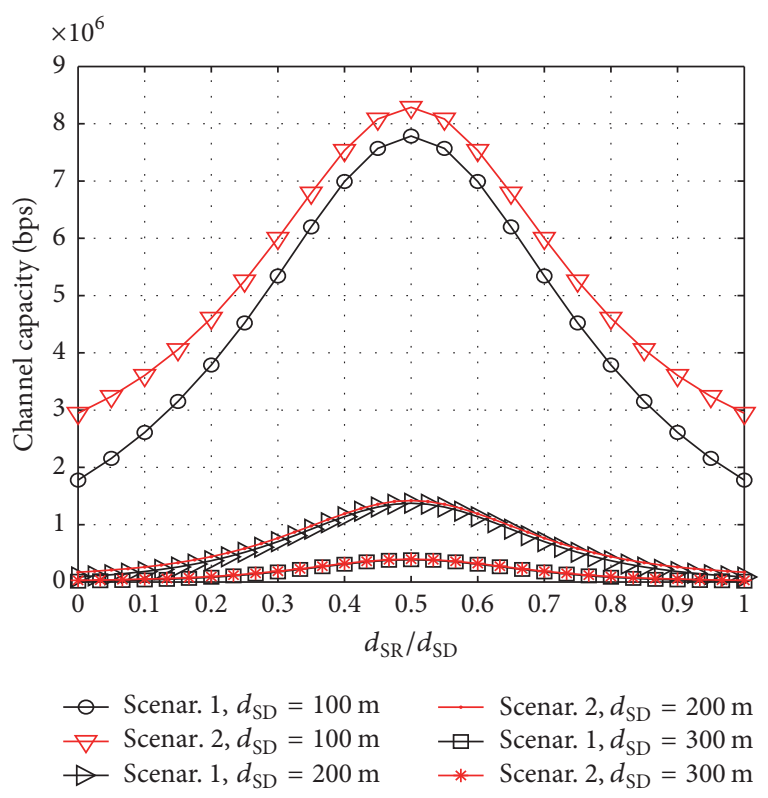

FIgURE 3: Channel capacity for OFDM-based two-hop relaying PLC-AF systems in the two scenarios, $P / \sigma^{2}=20 \mathrm{~dB}$.

the end-to-end SNR $\gamma_{\mathrm{D}}^{1, \mathrm{AF}}$ in (13) in the first scenario has a symmetric expression with respect to the SNR of S-R link $\left(\gamma_{\mathrm{SR}}\right)$ and the SNR of R-D link $\left(\gamma_{\mathrm{RD}}\right)$. Besides, the contribution of the direct link to the channel capacity performance is important when the communication distance decreases. This proves that the direct link, that is, scenario 2, does not lead to a significant enhancement in channel capacity compared to the first scenario, that is, without direct link, for the long communication distances.

Figure 4 illustrates the performance in terms of channel capacity for OFDM-based two-hop relaying PLC-DF system in the two scenarios and for various relay location ratios. We calculated the channel capacity for the same communication distance values, that is, $d_{\mathrm{SD}}=100 \mathrm{~m}, 200 \mathrm{~m}$, and $300 \mathrm{~m}$, and for $P / \sigma^{2}=20 \mathrm{~dB}$. It is clear that the maximal capacity is attained when the relay is amid the source and destination nodes, that is, $d_{\mathrm{SR}} / d_{\mathrm{SD}}=0.5$, for the two scenarios. This result stems from the fact that channel capacity in the first scenario (see (17)) is influenced by the harmful SNR between the S$\mathrm{R}$ link $\left(\gamma_{\mathrm{SR}}\right)$ and the R-D link $\left(\gamma_{\mathrm{RD}}\right)$. In the second scenario (see (18)), it depends on the worse SNR between the S-R link $\left(\gamma_{\mathrm{SR}}\right)$ and the sum of SNR on the R-D and S-D links $\left(\gamma_{\mathrm{SD}}+\right.$ $\left.\gamma_{\mathrm{RD}}\right)$. We also note, for OFDM-based two-hop relaying PLCDF system, from Figure 4 that the second scenario, that is, with S-D link, supplies an improvement for channel capacity compared to the first scenario especially when the relay node is near the source node, that is, $d_{\mathrm{SR}} / d_{\mathrm{SD}} \leq 0.5$, and for low communication distance values, that is, $d_{\mathrm{SD}}=100 \mathrm{~m}$.

From Figure 5, we note that when the effective SNR at the destination node $P / \sigma^{2}$ increases, the channel capacities improve for OFDM-based PLC-AF and PLC-DF systems in the two scenarios regardless of the communication distance. We note also that for a given communication distance the 


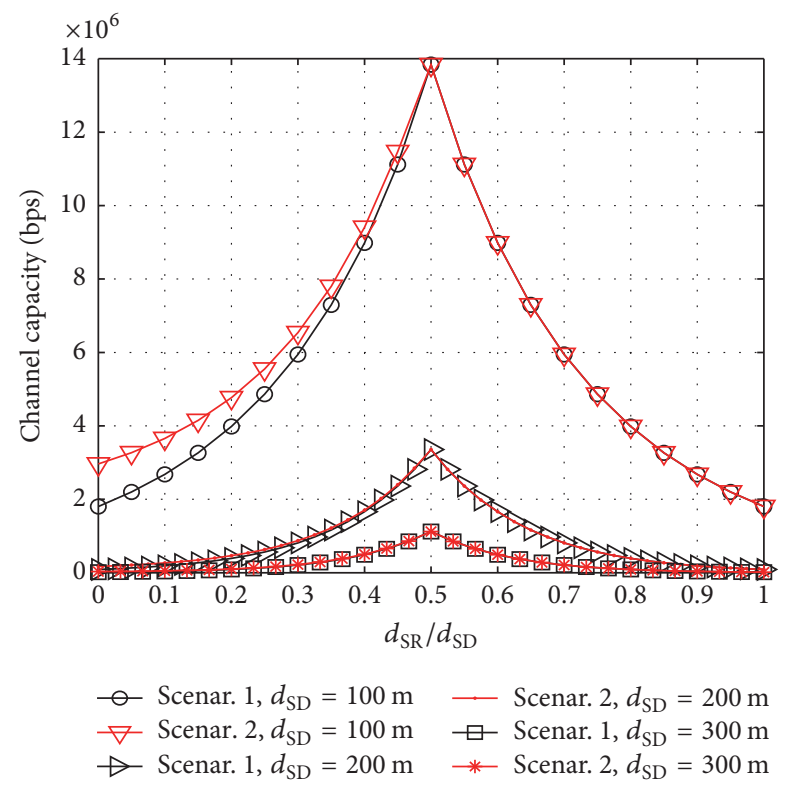

FIgURE 4: Channel capacity for OFDM-based two-hop relaying PLC-DF system in the two scenarios, $P / \sigma^{2}=20 \mathrm{~dB}$.

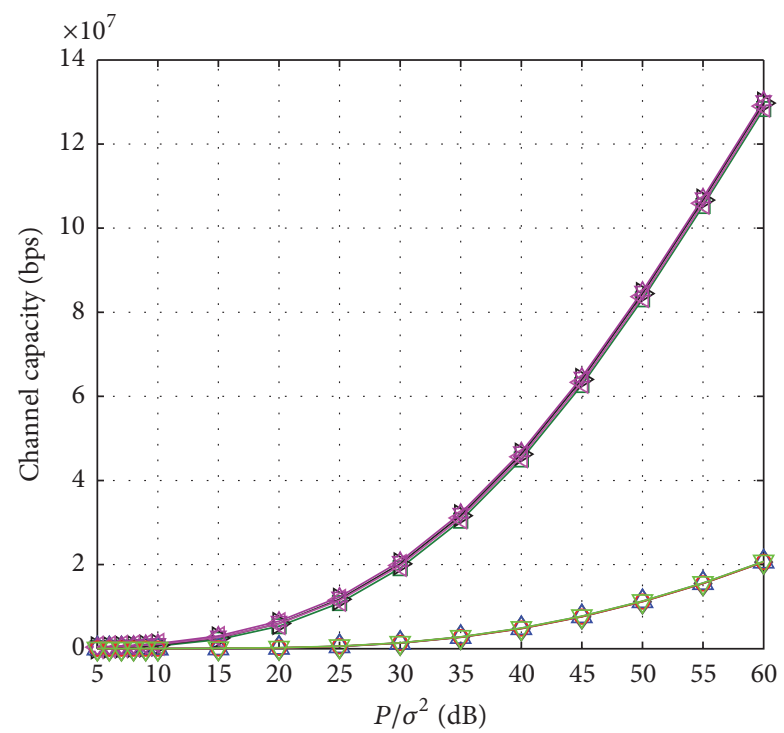

$$
\begin{array}{ll}
\square \text { Scr. } 1, \mathrm{AF}, d_{\mathrm{SD}}=100 \mathrm{~m} & \neg \text { Scr. 1, DF, } d_{\mathrm{SD}}=100 \mathrm{~m} \\
\neg \text { Scr. } 2, \mathrm{AF}, d_{\mathrm{SD}}=100 \mathrm{~m} & - \text { Scr. 2, DF, } d_{\mathrm{SD}}=100 \mathrm{~m} \\
\searrow \text { Scr. } 1, \mathrm{AF}, d_{\mathrm{SD}}=300 \mathrm{~m} & - \text { Scr. 1, DF, } d_{\mathrm{SD}}=300 \mathrm{~m} \\
\triangle \text { Scr. } 2, \mathrm{AF}, d_{\mathrm{SD}}=300 \mathrm{~m} & \nabla \text { Scr. 2, DF, } d_{\mathrm{SD}}=300 \mathrm{~m}
\end{array}
$$

FIGURE 5: Channel capacity for OFDM-based two-hop relaying PLC-AF and PLC-DF systems in the two scenarios when $d_{\mathrm{SD}}=$ $\{100 \mathrm{~m}, 300 \mathrm{~m}\}$.

channel capacity for OFDM-based PLC-DF and PLC-AF systems is very close.

Figure 6 represents the ABER for OFDM-based DL-PLC system when $d_{\mathrm{SD}}$ is equal to $100 \mathrm{~m}, 200 \mathrm{~m}$, and $300 \mathrm{~m}$ and when $P / \sigma^{2}$ varies. We remark that the ABER of the direct links is very bad especially when $d_{\mathrm{SD}}$ increases. Therefore, we are interested in analyzing the ABER in a cooperative

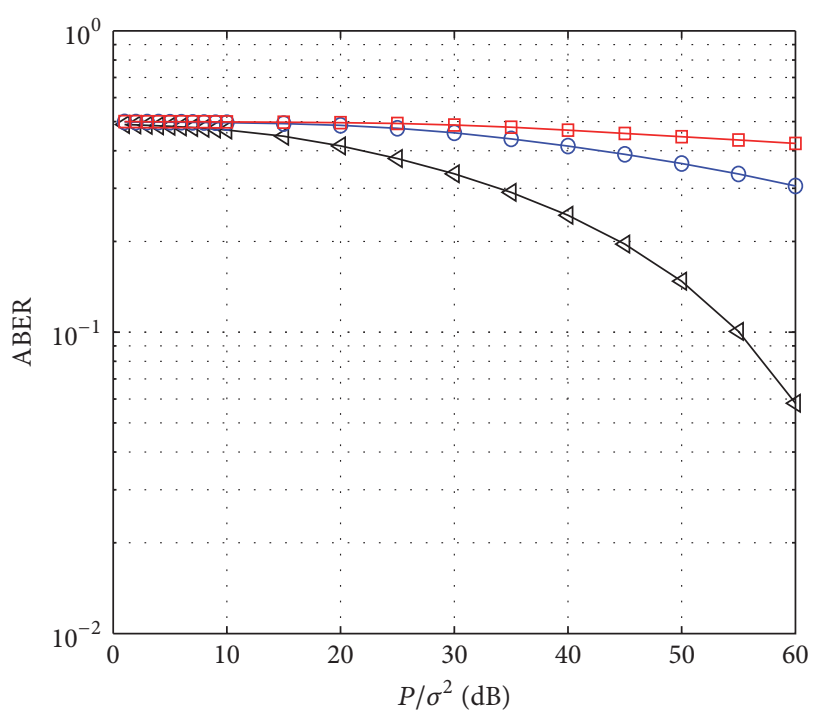

$$
\begin{aligned}
& \varangle d_{\mathrm{SD}}=100 \\
& -d_{\mathrm{SD}}=200 \\
& \square-d_{\mathrm{SD}}=300
\end{aligned}
$$

Figure 6: ABER for OFDM-based DL-PLC systems.

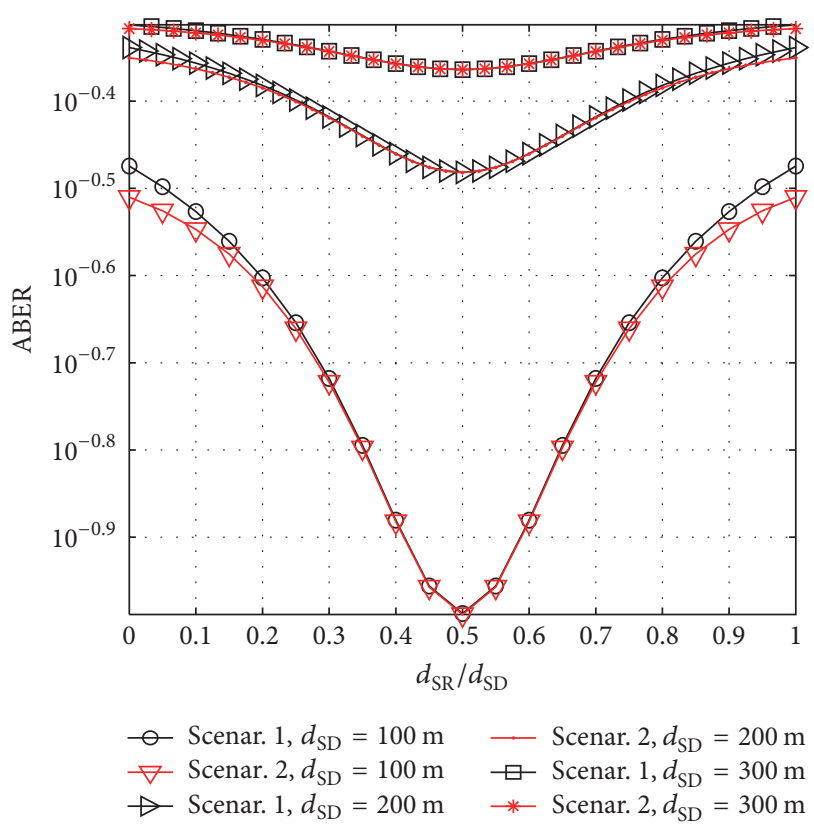

FIgUre 7: ABER for OFDM-based two-hop relaying PLC-AF systems in the two scenarios, $P / \sigma^{2}=30 \mathrm{~dB}$.

communication for the OFDM-based two-hop relaying PLCDF and PLC-AF systems.

Figure 7 shows the ABER performance in the two scenarios for OFDM-based two-hop relaying PLC-AF system when $d_{\mathrm{SD}}=100 \mathrm{~m}, 200 \mathrm{~m}, 300 \mathrm{~m}$ and $P / \sigma^{2}=30 \mathrm{~dB}$. We note that $\mathrm{ABER}$ performances are not significantly improved compared to direct link (see Figure 6) and particularly for low communication distance values, that is, $d_{\mathrm{SD}}=100 \mathrm{~m}$, and when the relay node is amid the source and destination nodes. 


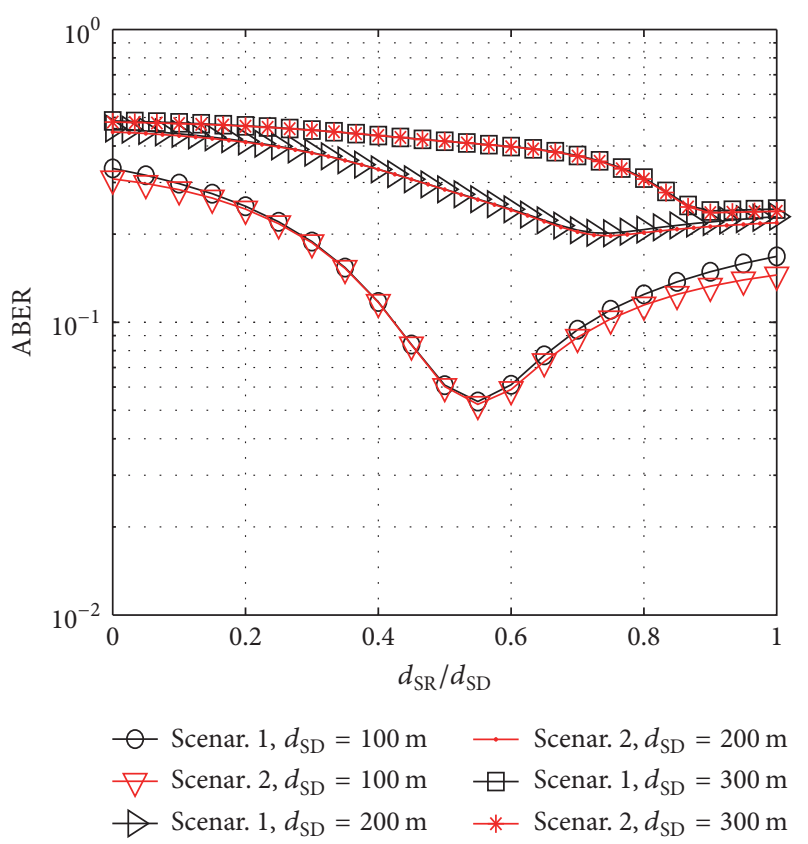

FIgURE 8: ABER for OFDM-based two-hop relaying PLC-DF systems for the two scenarios when $P / \sigma^{2}=30 \mathrm{~dB}$.

We also note that ABER performances in the two studied scenarios are very close.

ABER performances for OFDM-based two-hop relaying PLC-DF system for the two considered scenarios are presented in Figure 8. We note that the ABER performances are significantly improved compared to those reached for OFDM-based DL-PLC and two-hop relaying PLC-AF systems and they depend directly on the relay location, the considered scenario, and the communication distance. It is clear that the optimum relay location ratios depend on the communication distance values $d_{\mathrm{SD}}$; that is, for $d_{\mathrm{SD}}=$ $100 \mathrm{~m}, 200 \mathrm{~m}$, and $300 \mathrm{~m}$ when $P / \sigma^{2}=30 \mathrm{~dB}$ the optimal relay location ratios are approximately $0.55,0.75$, and 0.9 , respectively. We remark that the ABER performances are very close for the two considered scenarios.

From Figure 9, we note that when the effective SNR at the destination node $P / \sigma^{2}$ increases the ABER is improved dramatically for OFDM-based PLC-AF and PLC-DF systems in the two scenarios especially for low communication distance. We note also that for a given low communication distance, that is, $d_{\mathrm{SD}}=100 \mathrm{~m}$, the reached ABER for OFDMbased PLC-DF system is better than for PLC-AF system.

4.2. Performances Improvement. As aforementioned, since in the second scenario the destination node receives two versions of the original signal received from the source and the relay nodes during two time slots, we used at the destination node the MRC technique. The latter allows us to estimate the transmitted signal for OFDM-based two-hop relaying PLCAF and PLC-DF systems. According to the obtained simulation results, we noticed that the use of direct link, that is, scenario 2, did not significantly improve the channel capacity and ABER performances for the considered scenarios.

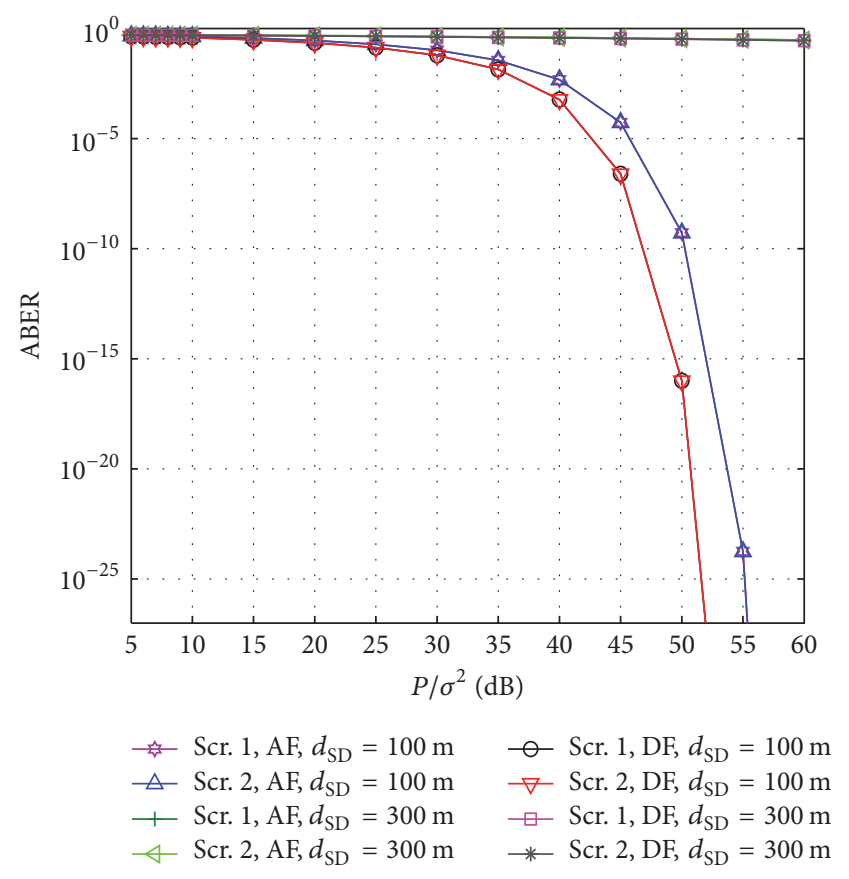

FIGURE 9: ABER for OFDM-based two-hop relaying PLC-AF and PLC-DF systems for the two scenarios when $d_{\mathrm{SD}}=\{100 \mathrm{~m}, 300 \mathrm{~m}\}$.

By analyzing the capacities expressions calculated in (16) and (18) and the ABER expressions in (22) and (30), we notice that the direct link, represented by $\gamma_{\mathrm{SD}}$ in the cited equations, directly affects the capacity and ABER performances. To optimize the use of direct link, we propose a solution based on data signal assignment indices permutation over the subcarriers. This solution aims to maximize the effective SNR calculated from the combined output signal at the destination node in order to improve the capacity and ABER. The choice of this solution is easily justified by the fact that PLC channel attenuation dramatically increases with frequency. By analyzing (16), (18), (22), and (30) and since the signal attenuation decreases linearly with frequency and communication distance (see Figure 10), the proposed solution aims to reduce these effects. Consequently, the proposed permutation principle is based on order inversion of the transmitted signals indices during the transmission over the direct link by the source node to the destination node. For instance, if data signal $x$ is transmitted on the $n$th subcarrier by the source node and the relay node over S-R, S-D, and R-D links during the first and second TS, respectively, according to the proposed solution this data signal $x$ will be transmitted on the $n$th and $(N-n+1)$ th subcarrier, over $\{S-R, R-D\}$ and S-D links, respectively. Consequently, the received signals during the second time slot from the source node on the $n$th subcarrier at the destination node in the second scenario for the OFDM-based two-hop relaying PLC-AF and PLC-DF systems have the following expression:

$$
\begin{aligned}
& y_{\mathrm{opt}, \mathrm{SD}}^{2, \mathrm{AF}}[n]=H_{\mathrm{SD}}[N-n+1] x[n]+n_{1, \mathrm{D}}[n], \\
& y_{\mathrm{opt}, \mathrm{SD}}^{2, \mathrm{DF}}[n]=H_{\mathrm{SD}}[N-n+1] x[n]+n_{1, \mathrm{D}}[n] .
\end{aligned}
$$




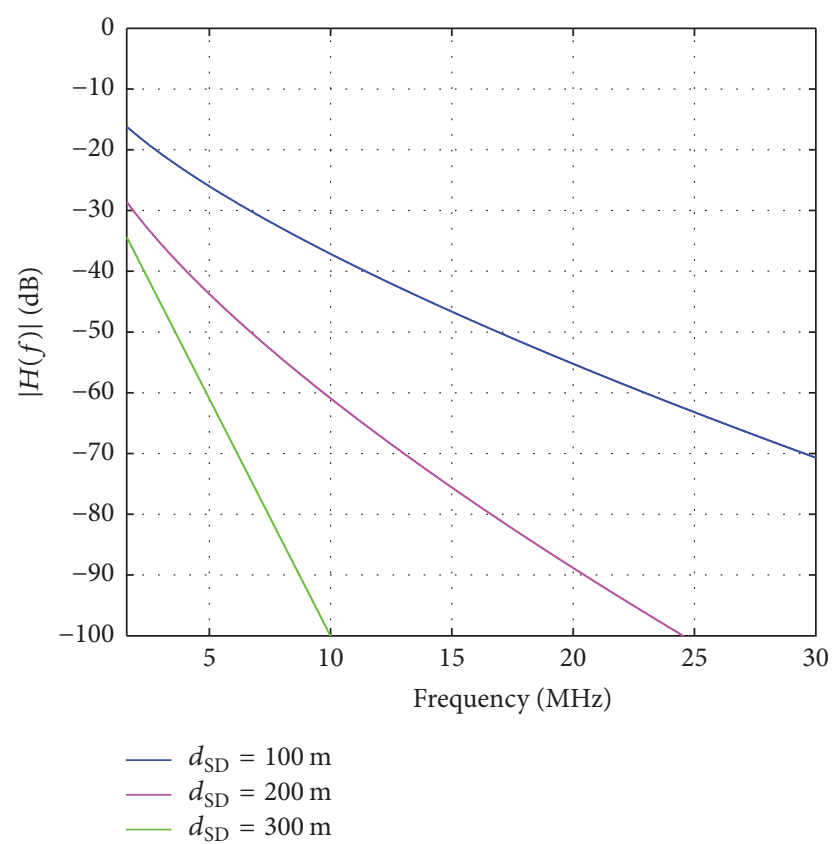

FIGURE 10: Impact of the distance on the signal attenuation.

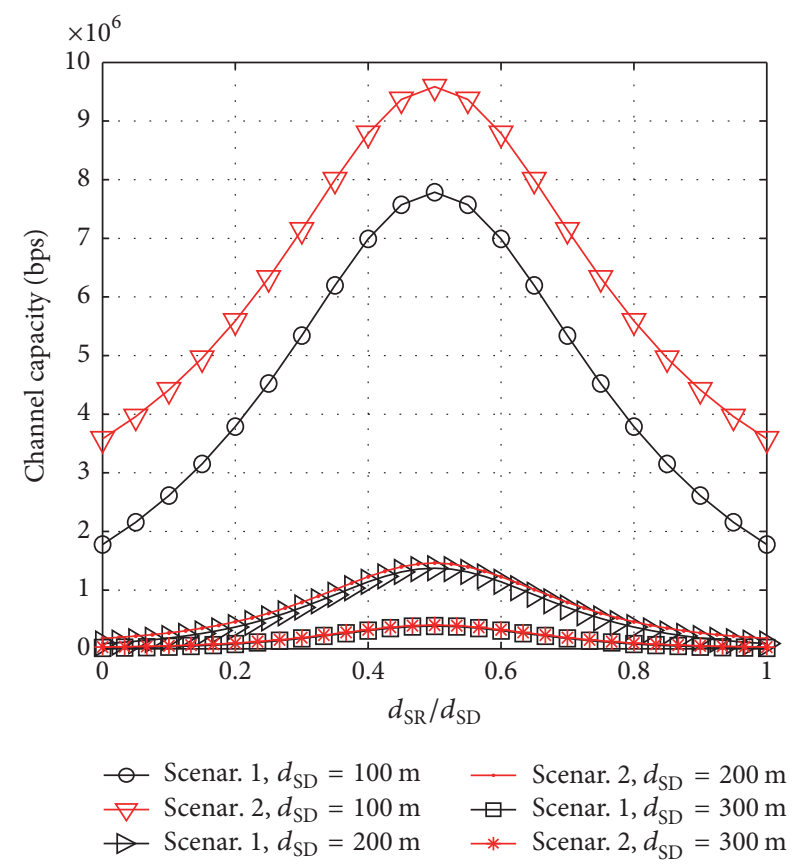

FIGURE 11: Channel capacity for OFDM-based two-hop relaying PLC-AF system for the two scenarios when $P / \sigma^{2}=20 \mathrm{~dB}$.

It results in the fact that the channel capacity and the ABER expressions on the $n$th subcarrier, with $(1 \leq n \leq N)$, for the OFDM-based two-hop relaying PLC-AF system are identical to $C^{2, \mathrm{AF}}[n]$ in (16) and $P^{2, \mathrm{AF}}[n]$ in (22), respectively. The maximum achievable capacity and ABER expressions for the OFDM-based two-hop relaying PLC-DF system at the $n$th subcarrier with $(1 \leq n \leq N)$ are identical to $C^{2, \mathrm{DF}}[n]$ in (18) and $P^{2, \mathrm{DF}}[n]$ in (30), respectively.

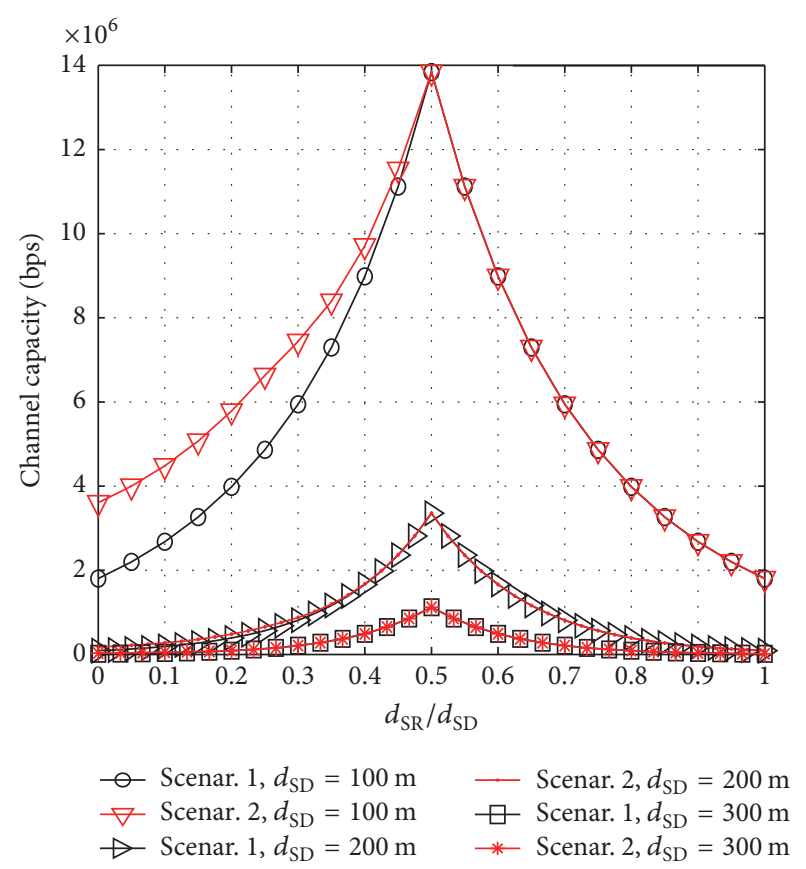

FIGURE 12: Channel capacity for OFDM-based two-hop relaying PLC-DF system for the two scenarios when $P / \sigma^{2}=20 \mathrm{~dB}$.

Figures 11 and 12 compare the channel capacity for the previously studied scenarios where in the second scenario we applied the proposed solution by permuting the subcarrier indices. We note that this proposed solution improves significantly the channel capacity for the second scenario especially for low communication distance values; that is, $d_{\mathrm{SD}}=100 \mathrm{~m}$ for OFDM-based two-hop relaying PLC-AF and PLC-DF systems.

We also notice an enhancement in the ABER performances when the improvement solution is used and this is illustrated in Figures 13 and 14. Accordingly, when we use the proposed optimization process the ABER performances are improved especially for the low communication distance values; that is, $d_{\mathrm{SD}}=100 \mathrm{~m}$ for OFDM-based two-hop relaying PLC-AF and PLC-DF systems.

Figure 15 illustrates the channel capacity for the previously studied scenarios, for OFDM-based two-hop relaying PLC-AF and PLC-DF systems, on which we applied the proposed solution for the second scenario where we permute the subcarrier indices. We note that this proposed solution significantly improves the channel capacity for the second scenario (compared to the illustrated results in Figure 5) especially for low communication distance values; that is, $d_{\mathrm{SD}}=100 \mathrm{~m}$ for OFDM-based two-hop relaying PLC-AF and PLC-DF systems.

From Figure 16 we note that the ABER performances are improved for the second scenario for OFDM-based two-hop relaying PLC-AF and PLC-DF systems when the improvement solution is presented. Additionally, it is clear that the ABER reached for OFDM-based two-hop relaying PLC-DF system are better than those for PLC-AF system. 


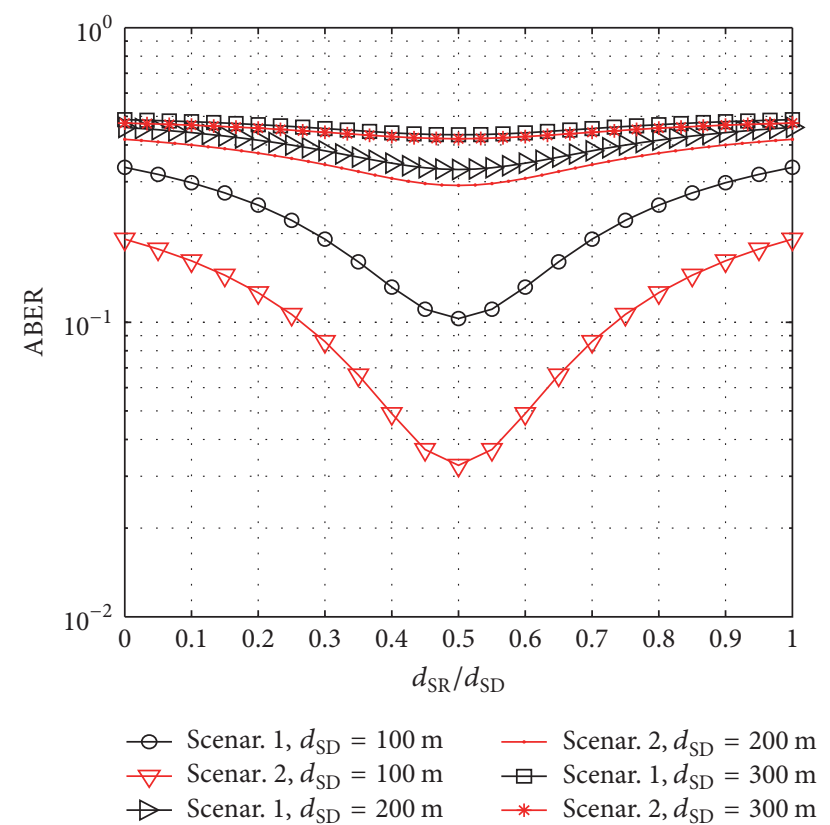

FIgURE 13: ABER for OFDM-based two-hop relaying PLC-AF system for the two scenarios when $P / \sigma^{2}=30 \mathrm{~dB}$.

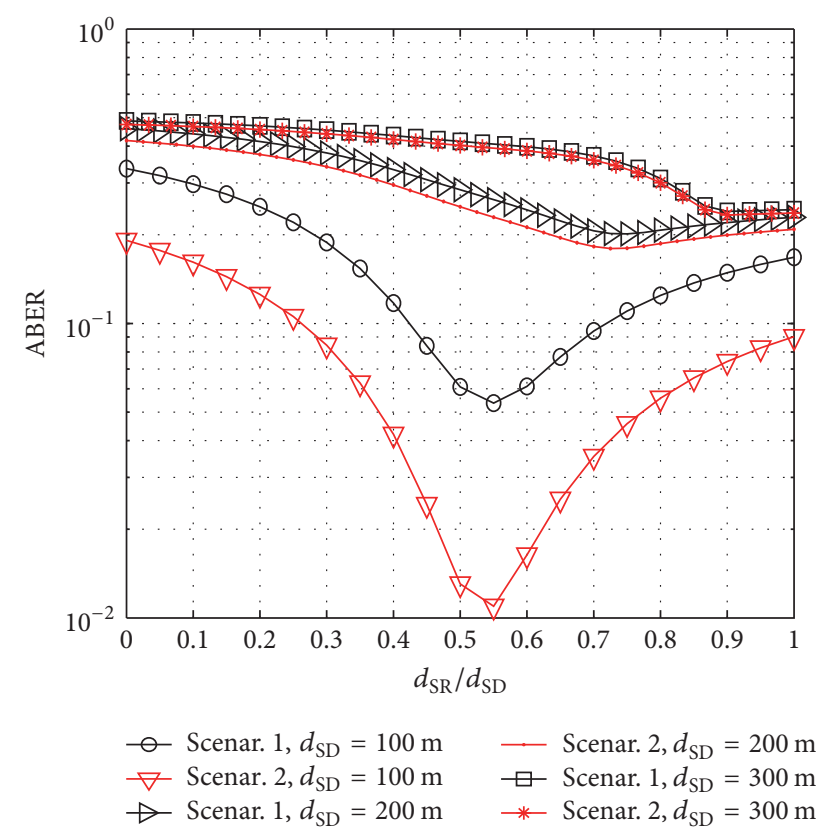

FIGURE 14: ABER for OFDM-based two-hop relaying PLC-DF system for the two scenarios when $P / \sigma^{2}=30 \mathrm{~dB}$.

\section{Conclusion}

In this paper, we derived the channel capacity and ABER expressions for OFDM-based two-hop relaying PLC-AF and PLC-DF systems for the two scenarios, that is, with and without direct link (DL), to study the performance in terms of channel capacity and ABER. We proved that the use of a relay halfway between two communicating nodes can drastically

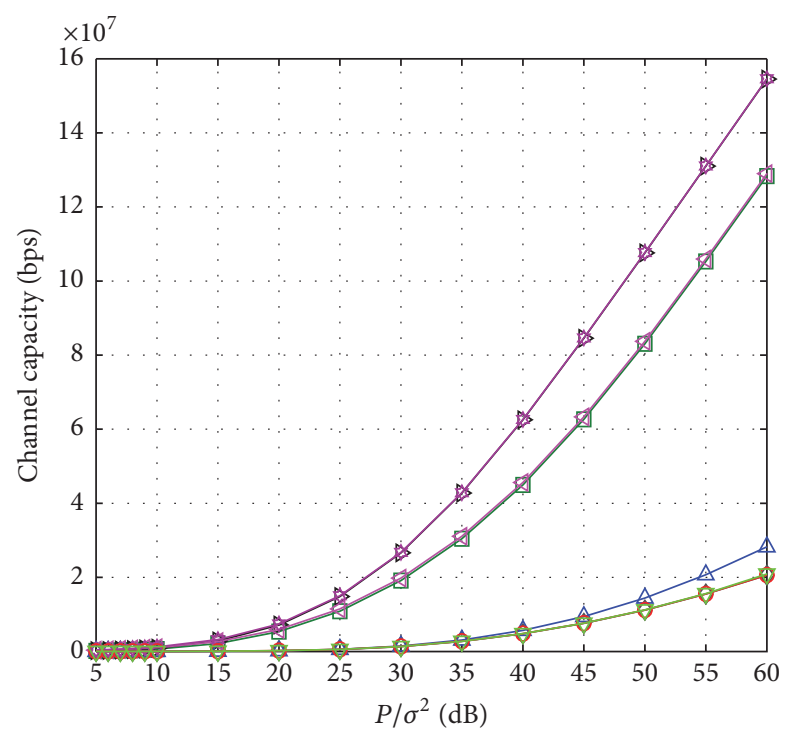

$$
\begin{array}{ll}
\square \text { Scr. } 1, \mathrm{AF}, d_{\mathrm{SD}}=100 \mathrm{~m} & \neg \text { Scr. } 1, \mathrm{DF}, d_{\mathrm{SD}}=100 \mathrm{~m} \\
\neg \text { Scr. } 2, \mathrm{AF}, d_{\mathrm{SD}}=100 \mathrm{~m} & \text { Scr. } 2, \mathrm{DF}, d_{\mathrm{SD}}=100 \mathrm{~m} \\
\searrow \text { Scr. } 1, \mathrm{AF}, d_{\mathrm{SD}}=300 \mathrm{~m} & - \text { Scr. } 1, \mathrm{DF}, d_{\mathrm{SD}}=300 \mathrm{~m} \\
\triangle \text { Scr. } 2, \mathrm{AF}, d_{\mathrm{SD}}=300 \mathrm{~m} & \nabla \text { Scr. 2, DF, } d_{\mathrm{SD}}=300 \mathrm{~m}
\end{array}
$$

FIGURE 15: Channel capacity for OFDM-based two-hop relaying PLC-AF and PLC-DF systems for the two scenarios when $d_{\mathrm{SR}} / d_{\mathrm{SD}}=$ 0.3 and $d_{\mathrm{SD}}=\{100 \mathrm{~m}, 300 \mathrm{~m}\}$.

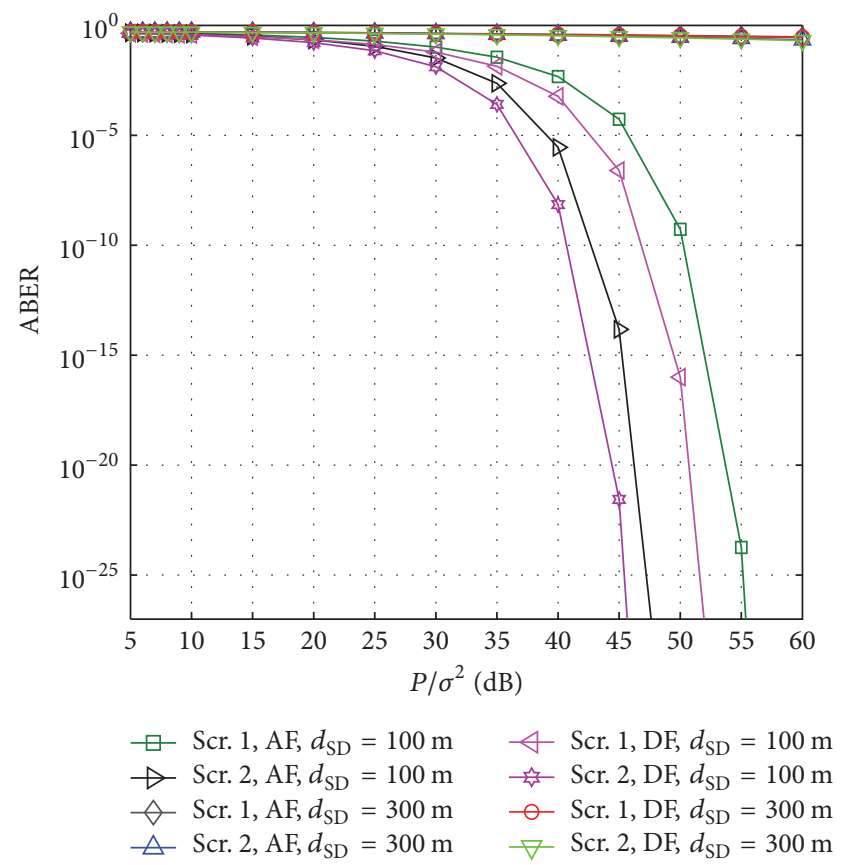

FIGURE 16: ABER for OFDM-based two-hop relaying PLC-AF and PLC-DF systems for the two scenarios when $d_{\mathrm{SR}} / d_{\mathrm{SD}}=0.5$ and $d_{\mathrm{SD}}=\{100 \mathrm{~m}, 300 \mathrm{~m}\}$.

improve the channel capacity and ABER performances compared to an OFDM-based DL-PLC system. We also proved that varying the relay location, between source node and destination node and the scenario, has a significant impact 
on the channel capacity and ABER performances. Numerical results demonstrated that the highest channel capacity is reached when the relay node is in the middle between the source and the destination nodes for OFDM-based two-hop relaying BB-PLC-AF and BB-PLC-DF systems for the proposed scenarios, which confirms our expectations. Moreover, we also note that the lowest ABER is achieved when the relay node is betwixt the source and destination nodes for OFDMbased two-hop relaying PLC-AF system in both scenarios. We noticed that the use of the direct link, that is, scenario 2 , does not improve significantly channel capacity and ABER performances. Therefore, we proposed a new original process to improve the use of direct link in order to maximize the effective SNR calculated from the combiner output signal at the destination node. This solution considerably improves the channel capacity and ABER performances especially for the low communication distance value. For future work, we will attempt to combine the studied protocols with adequate channel encoding to improve the channel capacity and ABER performances in realistic noise models.

\section{Competing Interests}

The authors declare that there are no competing interests related to this paper.

\section{References}

[1] H. C. Ferreira, L. Lampe, J. Newbury, and T. G. Swart, Power Line Communications: Theory and Applications for Narrowband and Broadband Communications over Power Lines, John Wiley \& Sons, Hoboken, NJ, USA, 2011.

[2] S. Galli, A. Scaglione, and Z. Wang, "For the grid and through the grid: the role of power line communications in the smart grid," Proceedings of the IEEE, vol. 99, no. 6, pp. 998-1027, 2011.

[3] M. Zimmermann and K. Dostert, "A multipath model for the powerline channel," IEEE Transactions on Communications, vol. 50, no. 4, pp. 553-559, 2002.

[4] H. Philipps, "Modeling of power line communications channels," in Proceedings of the IEEE International Symposium on Power Line Communications and Its Applications, pp. 14-21, 1999.

[5] M. Tlich, P. Pagani, G. Avril et al., "(PLC) channel characterization and modeling," Tech. Rep., OMEGA Project, 2008.

[6] P. Amirshahi and M. Kavehrad, "Transmission channel model and capacity of overhead multi-conductor mediumvoltage power-lines for broadband communications," IEEE Journal on Selected Areas in Communications, vol. 24, pp. 1292-1303, 2006.

[7] A. G. Lazaropoulos and P. G. Cottis, “Transmission characteristics of overhead medium-voltage power-line communication channels," IEEE Transactions on Power Delivery, vol. 24, no. 3, pp. 1164-1173, 2009.

[8] M. Zimmerman and K. Dostert, "An analysis of the broadband noise scenario in powerline networks," in Proceedings of the International Symposium on Powerline Communications and Its Application, pp. 131-138, 2000.

[9] Korean Standards Association, "Power line communications (PLC) high speed PLC medium access control (MAC) and physical layer (PHY), part 1: general requirements," Korean Industrial Standard KS X 4600-1, 2006.
[10] OPERA Technology White Paper, 2006, http://www.upaplc.org.

[11] J. N. Laneman, D. N. C. Tse, and G. W. Wornell, "Cooperative diversity in wireless networks: efficient protocols and outage behavior," IEEE Transactions on Information Theory, vol. 50, no. 12, pp. 3062-3080, 2004.

[12] A. Sendonaris, E. Erkip, and B. Aazhang, "User cooperation diversity. Part I. System description," IEEE Transactions on Communications, vol. 51, no. 11, pp. 1927-1938, 2003.

[13] X. Cheng, R. Cao, and L. Yang, "Relay-aided amplify-andforward powerline communications," IEEE Transactions on Smart Grid, vol. 4, no. 1, pp. 265-272, 2013.

[14] X. Cheng, R. Cao, and L. Yang, "On the system capacity of relayaided Powerline Communications," in Proceedings of the IEEE International Symposium on Power Line Communications and Its Applications (ISPLC '11), pp. 170-175, IEEE, Udine, Italy, April 2011.

[15] S. Ezzine, F. Abdelkefi, J. P. Cances, V. Meghdadi, and A. Bouallégue, "Joint network coding and OFDMA based MAClayer in PLC networks," in Proceedings of the 18th IEEE International Symposium on Power Line Communications and Its Applications (ISPLC '14), pp. 311-315, Glasgow, UK, April 2014.

[16] Y.-H. Kim, S. Choi, S.-C. Kim, and J.-H. Lee, "Capacity of OFDM two-hop relaying systems for medium-voltage powerline access networks," IEEE Transactions on Power Delivery, vol. 27, no. 2, pp. 886-894, 2012.

[17] A. G. Lazaropoulos and P. G. Cottis, "Capacity of overhead medium voltage power line communication channels," IEEE Transactions on Power Delivery, vol. 25, no. 2, pp. 723-733, 2010.

[18] J. Anatory, N. Theethayi, R. Thottappillil, M. M. Kissaka, and N. H. Mvungi, "Broadband power-line communications: the channel capacity analysis," IEEE Transactions on Power Delivery, vol. 23, no. 1, pp. 164-170, 2008.

[19] L. Lampe and A. J. H. Vinck, "On cooperative coding for narrow band PLC networks," AEU-International Journal of Electronics and Communications, vol. 65, no. 8, pp. 681-687, 2011.

[20] S. Galli and T. C. Banwell, "A novel approach to the modeling of the indoor power line channel-part II: transfer function and its properties," IEEE Transactions on Power Delivery, vol. 20, no. 3, pp. 1869-1878, 2005.

[21] D. G. Brennan, "Linear diversity combining techniques," Proceedings of the IEEE, vol. 91, no. 2, pp. 331-356, 2003.

[22] P. Merkey and E. C. Posner, "Optimum cyclic redundancy codes for noisy channels," IEEE Transactions on Information Theory, vol. 30, pp. 865-867, 1984.

[23] J. G. Proakis, Digital Communications, McGraw-Hill, New York, NY, USA, 4th edition, 2001. 


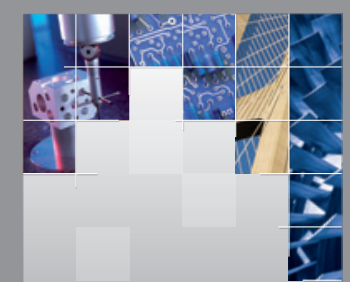

\section{Enfincering}
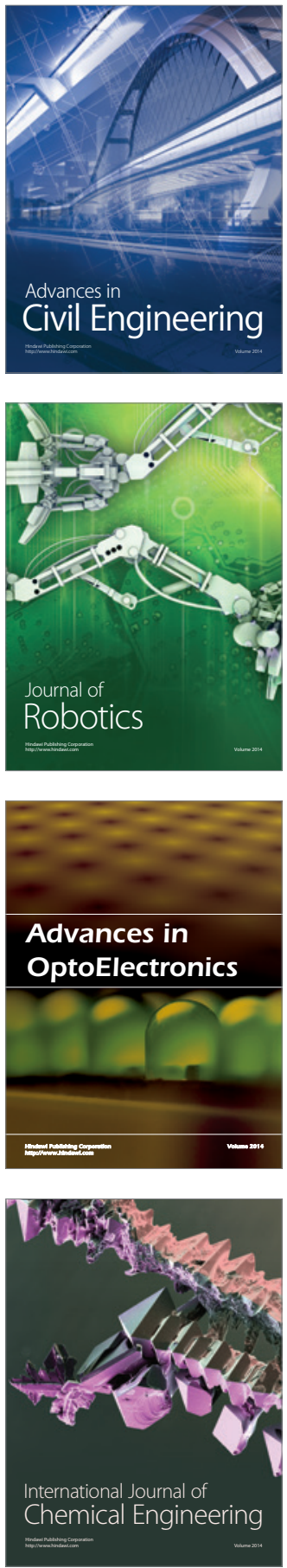

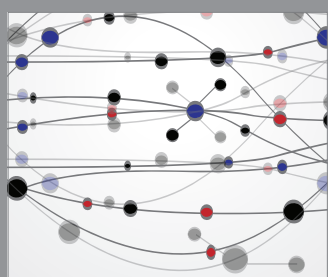

The Scientific World Journal

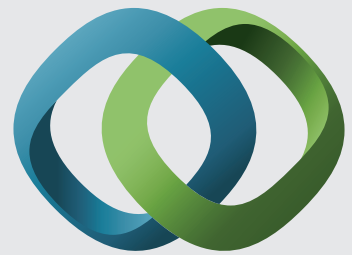

\section{Hindawi}

Submit your manuscripts at

https://www.hindawi.com
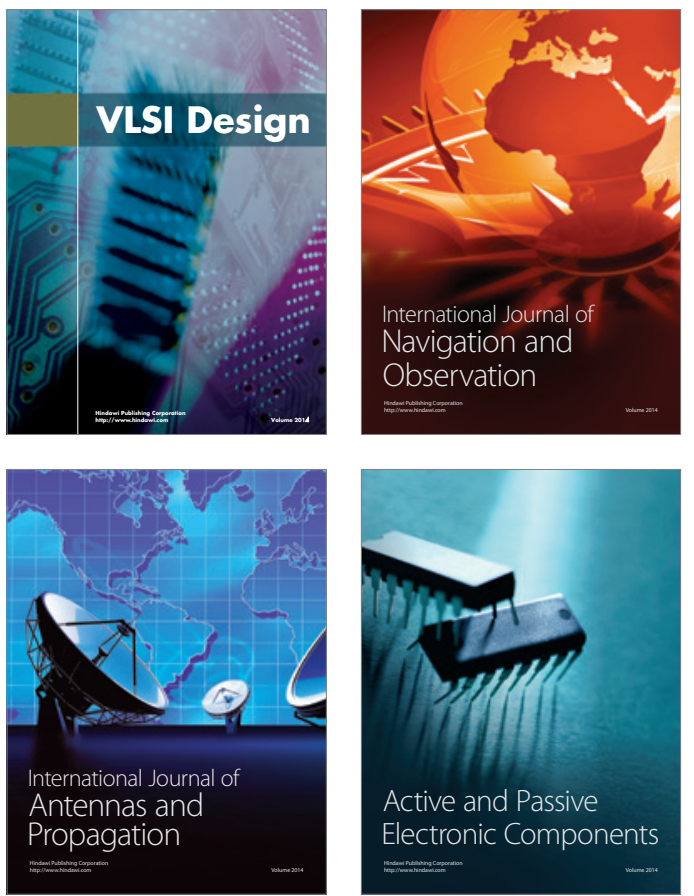
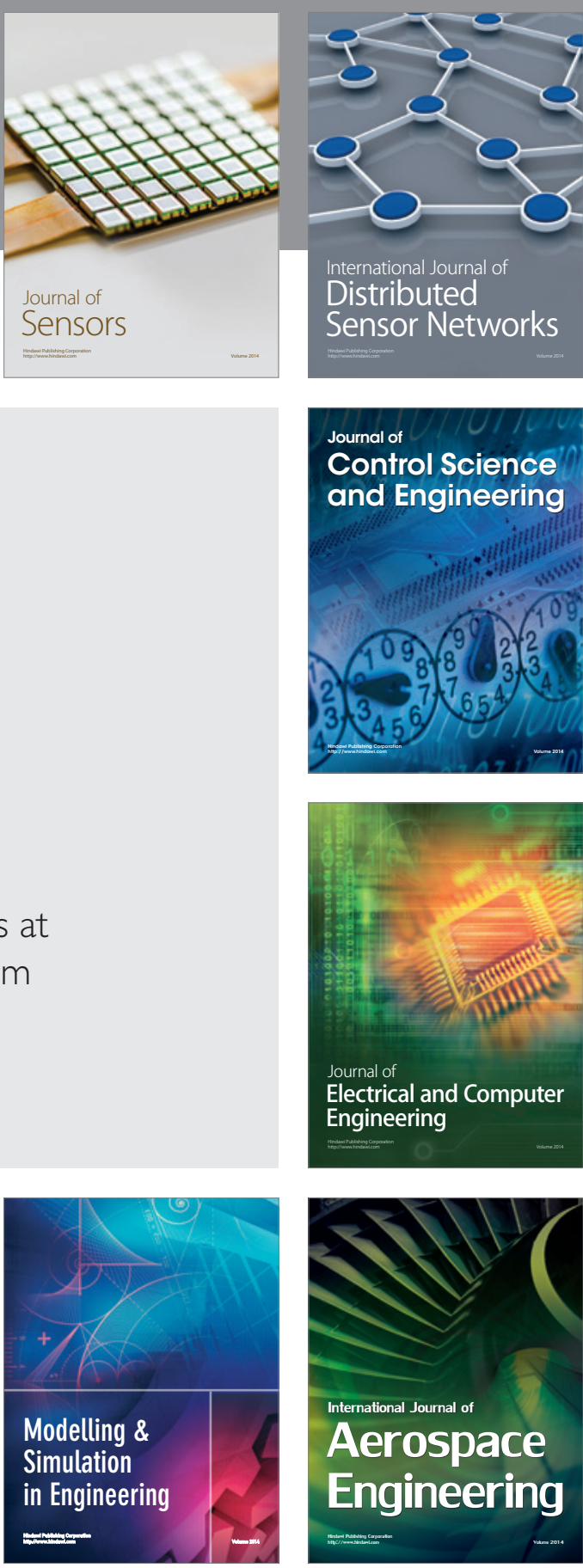

International Journal of

Distributed

Sensor Networks

$-$

Joumal of

Control Science

and Engineering
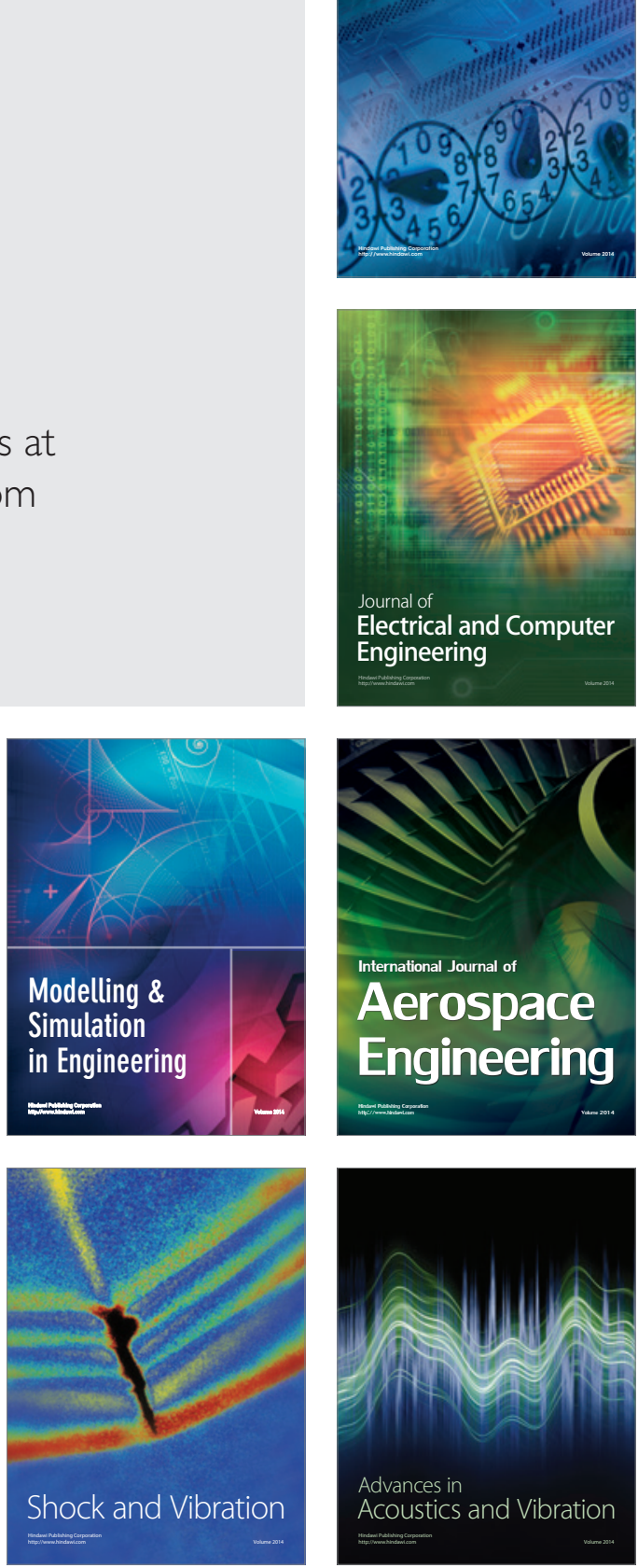